\title{
Nevo melanocítico congênito: estudo retrospectivo dos aspectos epidemiológicos e terapêuticos em uma série de 45 pacientes
}

\author{
Congenital melanocytic nevi: a retrospective study of epidemiological and \\ therapeutic aspects in a series of 45 patients
}

\author{
Gladstone Eustaquio de \\ LIMA FARIA ${ }^{1}$ \\ Dov Charles Goldenberg ${ }^{2}$ \\ Tatiana de Moura ${ }^{3}$ \\ Patrícia Yuko Hiraki ${ }^{4}$ \\ Rafael Andrade Manzini ${ }^{5}$ \\ Eduardo Soares Cirne de \\ TOLEDO $^{6}$ \\ Marcus Castro Ferreira ${ }^{7}$
}

Trabalho realizado na Divisão de Cirurgia Plástica e Queimaduras do Hospital das Clínicas da Faculdade de Medicina da Universidade de São Paulo, São Paulo, SP, Brasil.

Artigo submetido pelo SGP (Sistema de Gestão de Publicações) da RBCP.

Artigo recebido: 24/1/2011 Artigo aceito: 23/3/2011

\section{RESUMO}

Introdução: Os nevos melanocíticos congênitos, apesar de apresentarem uma incidência relativamente rara, são lesões que necessitam acompanhamento por longo período, a fim de se evitar sequelas psicológicas e complicações inerentes a essa patologia, como estresse psicológico, melanose neurocutânea e degeneração maligna. Método: O presente estudo demonstra os dados epidemiológicos e terapêuticos em uma série de 45 casos atendidos na Divisão de Cirurgia Plástica e Queimaduras do Hospital das Clínicas da Faculdade de Medicina da Universidade de São Paulo.

Descritores: Nevos e Melanomas. Nevo. Melanoma. Melanose.

\begin{abstract}
Introduction: Congenital melanocytic nevi despite presenting a relatively rare incidence are injuries that require monitoring for a long period. The purpose of this is to prevent psychological sequelae and complications inherent to this pathology, such as psychological stress, neurocutaneous melanosis and malignant degeneration. Methods: The present study demonstrates the epidemiological data and treatment in a series of 45 cases treated in the Divisão de Cirurgia Plástica e Queimaduras do Hospital das Clínicas da Faculdade de Medicina da Universidade de São Paulo (Division of Plastic Surgery and Burns of School of Medicine, University of São Paulo).
\end{abstract}

Keywords: Nevi and Melanomas. Nevus. Melanoma. Melanosis.

\footnotetext{
1. Pós-graduação em Cirurgia Plástica em andamento na Faculdade de Medicina da Universidade de São Paulo (FMUSP); Médico residente em Cirurgia Plástica pela Divisão de Cirurgia Plástica e Queimaduras do Hospital das Clínicas da Faculdade de Medicina da Universidade de São Paulo (HCFMUSP), São Paulo, SP, Brasil.

2. Professor livre docente do Departamento de Cirurgia da FMUSP; Médico assistente da Divisão de Cirurgia Plástica e Queimaduras do HCFMUSP, São Paulo, SP, Brasil.

3. Mestre em Cirurgia Plástica; Médica colaboradora da Divisão de Cirurgia Plástica e Queimaduras do HCFMUSP, São Paulo, SP, Brasil.

4. Especialista em Cirurgia Plástica; Médica colaboradora da Divisão de Cirurgia Plástica e Queimaduras do HCFMUSP, São Paulo, SP, Brasil.

5. Residência em Cirurgia Geral pelo HCFMUSP, São Paulo, SP, Brasil.

6. Aluno de graduação em Medicina pela FMUSP, São Paulo, SP, Brasil.

7. Professor titular da Divisão de Cirurgia Plástica e Queimaduras do HCFMUSP, São Paulo, SP, Brasil.
} 


\section{INTRODUÇÃO}

Os nevos melanocíticos congênitos (NMC) são lesões pigmentadas presentes ao nascimento, decorrentes do acúmulo de melanócitos de origem neuroectodérmica em localização ectópica ${ }^{1}$. Algumas características histológicas peculiares são capazes de definir a etiologia congênita do nevo, tais como a presença de células névicas agrupadas em cachos e a presença destes elementos celulares em estruturas glandulares, vasos sanguíneos e tecido subcutâneo ${ }^{2}$.

A incidência de NMC na população geral é de 1:100 nascidos vivos e, quando se consideram apenas os nevos gigantes, a incidência se reduz para 1:20.000 nascidos vivos ${ }^{3}$. Sua distribuição corporal é ampla, sendo localizados com maior frequência no tronco e na face ${ }^{4,5}$.

NMC são classificados quanto as suas dimensões de diversas maneiras. As três classificações mais citadas levam em conta o maior diâmetro do nevo, sua área ou a porcentagem corporal que ocupam. São considerados nevos gigantes quando, respectivamente para cada classificação, apresentam mais que $20 \mathrm{~cm}$ de diâmetro ou compreendem área corpórea superior a $100 \mathrm{~cm}^{2}$ ou maior que $2 \%$ da superfície corporal ${ }^{6-8}$.

A presença de melanose neurocutânea, a degeneração maligna para melanoma e o estresse psicológico para familiares e para a criança são as principais motivações para o tratamento do $\mathrm{NMC}^{9}$.

A incidência real de melanoma em NMC permanece controversa na literatura e casuísticas mais recentes apresentam dados que variam entre 2,8 e $8,5 \%$ dos portadores de nevos congênitos ${ }^{4}$. Tamanho do nevo acima de $20 \mathrm{~cm}^{2}$, número de lesões e faixa etária menor que três anos são as características clínicas mais associadas ao risco de transformação maligna ${ }^{7,10-14}$.

O tratamento cirúrgico é a opção preferencial, quando indicado. Entre as modalidades cirúrgicas merecem destaque a ressecção seguida de enxertia de pele, ressecções parceladas e o uso dos expansores teciduais nas áreas sãs adjacentes, seguido de ressecção das lesões e avanço ou rotação dos retalhos expandidos.

O objetivo do presente trabalho é demonstrar a experiência de um serviço de referência para o tratamento de NMC do ponto de vista epidemiológico, apresentação clínica, modalidade cirúrgica empregada, relatórios histopatológicos e o índice de malignização em uma série de 45 pacientes.

\section{MÉTODO}

Foi realizado estudo retrospectivo por meio de revisão de prontuário de pacientes atendidos na Divisão de Cirurgia Plástica e Queimaduras do Hospital das Clínicas da Faculdade de Medicina da Universidade de São Paulo, no período compreendido entre 1998 e 2010 . Foi definido o diagnóstico de nevo melanocítico congênito, considerando-se os códigos do CID-10 relacionados D-28, I-78.1 e Q-82.5. Foram localizadas e analisadas as informações de 45 prontuários de pacientes.

Foram avaliados dados epidemiológicos, clínicos e anatomopatológicos. Dados epidemiológicos incluíram idade, gênero, etnia e histórico familiar. Clinicamente foi analisada a localização das lesões, bem como suas dimensões e a presença de lesões satélites. Avaliada a modalidade cirúrgica empregada, o número de abordagens cirúrgicas necessárias, além de suas complicações.

Foi realizada, também, revisão dos estudos anatomopatológico das peças ressecadas, observando-se os tipos histológicos, o tamanho macroscópico da peça cirúrgica e incidência de focos neoplásicos.

\section{RESULTADOS}

Dos 45 pacientes que foram avaliados, $62 \%$ eram do sexo feminino e apenas $13 \%$ apresentavam antecedente familiar de nevo congênito. Quanto à distribuição étnica, $75 \%$ dos pacientes eram da raça branca, $16 \%$ pardos, $5 \%$ negros e $4 \%$ amarelos (Tabela 1).

A localização mais frequente dos NMC foi a região de cabeça e pescoço, em $35 \%$ dos casos, seguida de tronco com $27 \%$ e membros com $9 \%$. O acometimento simultâneo em dois segmentos corporais ocorreu em $20 \%$ dos pacientes e, em $9 \%$ deles, três ou mais segmentos estavam acometidos (Figura 1).

Com relação à dimensão dos nevos, $69 \%$ dos casos apresentaram superfície corporal maior ou igual a $2 \%$, caracterizando, portanto, nevo congênito gigante. Excluindo-se os nevos menores que $1 \%$ de superfície corporal, a média aritmética do acometimento das lesões foi de 9,65\% (2-50\%).

Lesões satélites estavam presentes em $30 \%$ dos casos estudados.

\begin{tabular}{l|c}
\hline \multicolumn{2}{c}{ Tabela 1 - Dados epidemiológicos de pacientes portadores de } \\
NMC.
\end{tabular}




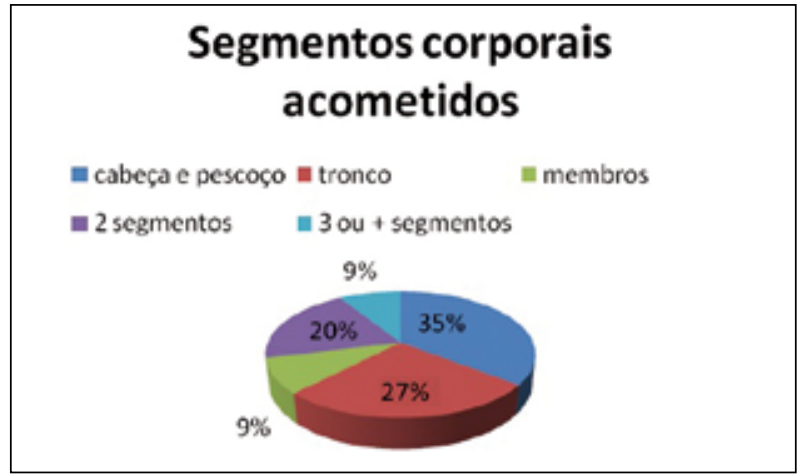

Figura 1 - Distribuição dos pacientes portadores de NMC segundo segmentos corporais acometidos.

$\mathrm{Na}$ presente casuística, 29 (64\%) pacientes foram submetidos a algum tipo de tratamento cirúrgico. Os 16 casos restantes encontram-se sob observação clínica, recusaram o tratamento cirúrgico ou ainda aguardam programação cirúrgica.

Em números absolutos, nesses 29 pacientes cirúrgicos, foram realizadas 66 ressecções parceladas, 13 ressecções seguidas de enxertia de pele e 18 expansões teciduais seguidas de rotação de retalhos expandidos (Tabela 2). Cada criança recebeu, em média, 2,57 abordagens cirúrgicas (mínimo de 1 e máximo de 9), sendo que apenas 7 casos receberam alta médica com tratamento concluído. Os demais pacientes aguardam pequenas ressecções, recusaram a continuidade do tratamento devido à grande exposição dos pacientes aos procedimentos cirúrgicos ou ainda devido a perda de seguimento.

Foram consideradas complicações do tratamento, infecções de ferida operatória ou expansor, pequenas deiscências, distúrbios de cicatrização, dentre outros. Tivemos registro de $31 \%$ de complicações, sendo a maior parte delas infecciosas (4/29). Outras complicações foram pequenas deiscências de ferida operatória $(2 / 29)$, perfuração de expansores (2/29) e cicatriz hipertrófica (1/29) (Tabela 2).

A análise dos resultados anatomopatológicos revelou que, em 20 casos, havia relato de nevos compostos, 12 de nevos intradérmicos e 2 nevos juncionais. Desse montante, 16 dos relatórios mencionaram características patognomônicas de padrão congênito nos mesmos. Foram evidenciados, ainda, pacientes com diagnóstico de nevo azul, nevo sebáceo de Jadassohn e hamartoma de cisto neural, além de casos isolados de nódulo proliferativo em nevo congênito, hiperplasia típica e melanoma / padrão Spitzoide.

No presente estudo, apenas um $(2,2 \%)$ dos pacientes teve o diagnóstico de melanoma. Apesar do laudo revisado ter aventado o diagnóstico diferencial de padrão

\begin{tabular}{l|c}
\hline \multicolumn{2}{c}{ Tabela 2 - Dados cirúrgicos de pacientes portadores } \\
de NMC.
\end{tabular}

Spitzoide, foi considerado diagnóstico de melanoma para fins de tratamento e acompanhamento do caso. Tratava-se de paciente do sexo feminino, branca, lesão localizada em tronco e ocupando $24 \%$ da superfície corporal, sem lesões satélites ou melanose neurocutânea. Para seu tratamento, já foram realizadas 7 abordagens cirúrgicas, sendo 5 ressecções parceladas e 2 expansões teciduais.

A melanose neurocutânea foi afecção presente em 3 $(6,67 \%)$ casos, em localização meníngea, subcutânea e ungueal. Todos os pacientes eram pacientes brancos, com nevos de localização corporal multisegmentar e somente um deles apresentava lesões satélites.

Ainda em relação aos espécimes ressecados, foi observado que, em média, cada paciente teve $147,4 \mathrm{~cm}^{2}$ de pele ressecada, sendo o caso de maior ressecção totalizado em $560 \mathrm{~cm}^{2}$.

As Figuras 2 a 5 ilustram alguns casos dessa casuística.

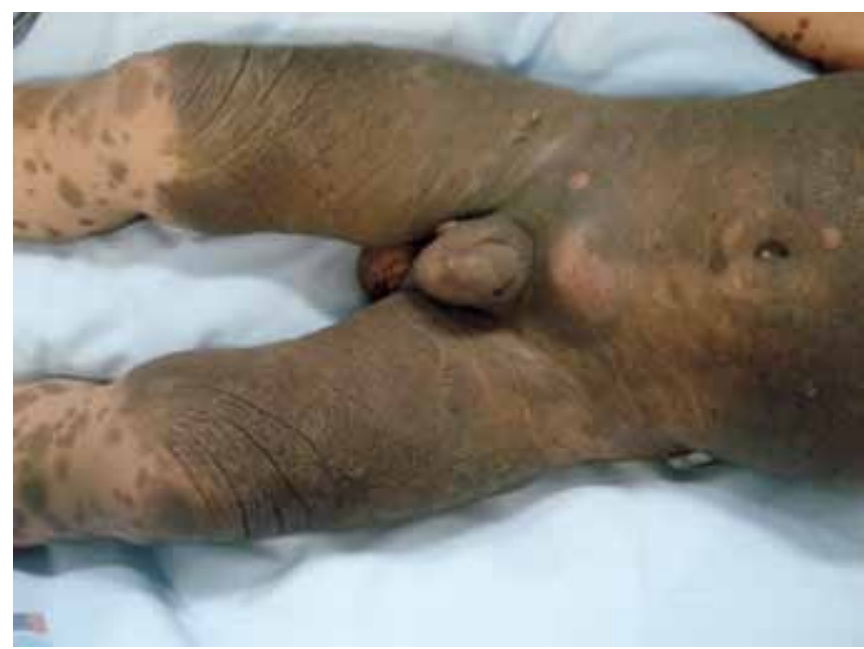

Figura 2 - Paciente portador de NMC e melanose neurocutânea demonstrando nevo ocupando $50 \%$ da superficie corporal. 


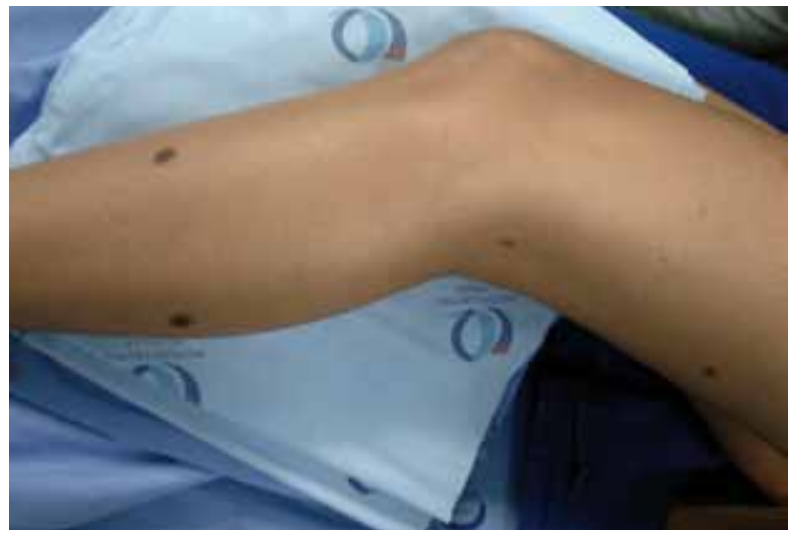

Figura 3 - Foto ilustrativa de lesões satélites em NMC.

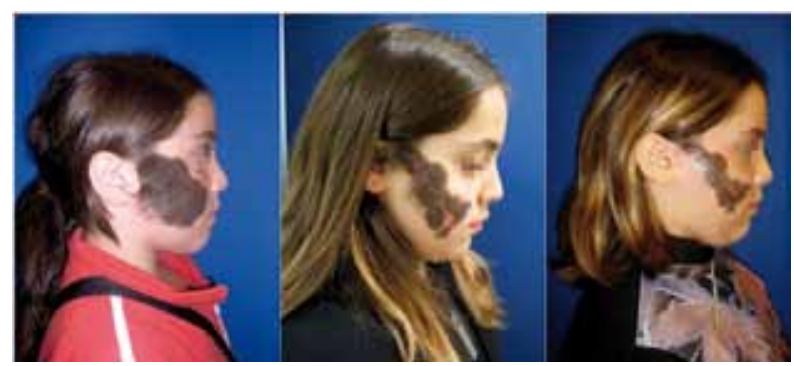

Figura 4 - Exemplo de paciente com nevo congênito em face submetida a ressecções parceladas.

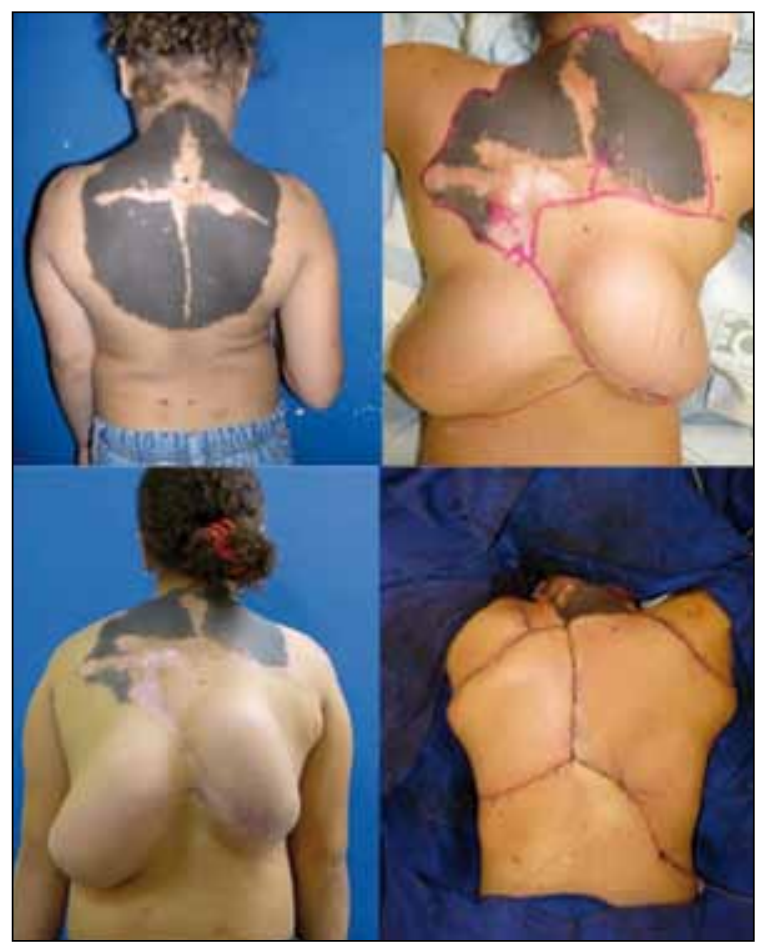

Figura 5 - Exemplo de expansão tecidual e rotação de retalho em nevo congênito gigante em dorso.

\section{DISCUSSÃO}

Os nevos congênitos, apesar de apresentarem incidência relativamente rara, são lesões que necessitam acompanhamento multidisciplinar por longo período, a fim de se evitar sequelas psicológicas e complicações inerentes a essa patologia.

$\mathrm{Na}$ casuística apresentada, predominaram os pacientes pediátricos do sexo feminino, não sendo encontrado na literatura consenso com relação ao gênero mais frequente. Com relação à etnia, os dados corroboram com prevalência maior na raça branca.

Os dados encontrados apontam a região da cabeça e pescoço como mais frequente para NMC, corroborando com os resultados publicados por Turkmen et al. ${ }^{5}$, porém outros estudos acusam o tronco como sítio mais frequente ${ }^{15}$. Cabe ressaltar que, no presente estudo, grande parcela dos nevos atingiam dimensões que ultrapassam os limites dos segmentos corporais, perfazendo um total de $29 \%$ dos casos com mais de 2 segmentos acometidos.

Arneja e Gosain ${ }^{2}$ publicaram dados que demonstram a prevalência de $80 \%$ de lesões satélites aos NMC, o que contraria os resultados aqui apresentados, que apontam apenas $30 \%$ dos casos.

É consenso que o tratamento cirúrgico seja o mais eficaz quando pertinente sua indicação, porém a tática cirúrgica depende das características da lesão, da complexidade do serviço e da experiência pessoal do cirurgião plástico. A conduta utilizada neste estudo vai de encontro à proposta da literatura internacional, com predominância de ressecção parcelada, seguida de expansão tecidual ${ }^{5}$. A razão que explica essa prevalência talvez seja a facilidade de execução do primeiro método, com menores custos e taxas de morbidade, porém exigindo maior número de abordagens para resolução adequada do caso, quando comparado à expansão tecidual.

As complicações infecciosas são maiores em pacientes pediátricos e, especificamente com expansores, a infecção permanece como o principal tipo de complicação. A incidência varia em torno de 4,4 a $9,3 \%$ dos $\operatorname{casos}^{16-21}$. Nossa casuística revelou incidência de $13 \%$ e, conforme já proposto na literatura, a infecção não indica a suspensão do programa de expansão, bem como o insucesso do mesmo. Em contrapartida, a extrusão é outra complicação importante e requer a retirada precoce do expansor ${ }^{21}$.

A degeneração maligna para melanoma é considerada complicação importante dos NMC, em decorrência da elevada morbi-mortalidade dessa patologia. Trabalhos iniciais publicados demonstravam variada incidência de melanomas, com estudos demonstrando incidências de zero a $42 \%{ }^{22-24}$. Estudos recentes têm demonstrado que a incidência é menor do que se acreditava, em torno de 2,8 a $8,5 \%$ dos $\mathrm{NMC}^{7,9}$. Os resultados obtidos estão de acordo com a literatura atual, visto que obtivemos incidência de apenas $2,2 \%$. Apesar da identificação de somente um caso de degeneração maligna em nossa casuística, podemos observar que o caso 
em questão apresentava os fatores de risco propostos na literatura: tamanho elevado (no caso $24 \%$ de superfície corporal) e idade menor que três anos ${ }^{4,25}$.

Obedecendo aos critérios diagnósticos mais recentes para a melanose neurocutânea, 3 pacientes apresentaram melanose neurocutânea em nossa casuística. Apesar de ambos nevos volumosos, apenas 1 apresentava nevos satélites, considerado importante fator de risco para melanose neurocutânea, principalmente quando apresenta mais de 20 lesões satélites ${ }^{26}$. Localização na linha média e em cabeça e pescoço também têm sido considerados fatores de risco para a melanose neurocutânea ${ }^{27}$, o que não foi observado em nossa casuística. Em ambos os casos, a lesão era substancialmente volumosa para as considerarmos como restrita a algum segmento corporal ou em linha média do corpo.

A melanose neurocutânea, a depender da idade de incidência, pode cursar com manifestações clínicas, como graus variados de retardo do desenvolvimento neuropsicomotor, crises convulsivas, restrição de crescimento pondero-estatural, dentre outras.

Neste estudo retrospectivo, pudemos observar preferência pelo tratamento de pacientes portadores de nevos de grandes dimensões, que requerem grande número de abordagens cirúrgicas e seguimento. O seguimento clínico é peça fundamental nesse tipo de paciente, a fim de diagnosticar precocemente malignidades e outras complicações.

Os pacientes portadores de NMC gigante merecem especial atenção por equipe multiprofissional, incluindo terapeutas ocupacionais, psicoterapeutas e cirurgiões plásticos, que geralmente conduzem os casos. A sequela psicológica a esses pacientes ocorrem tanto pelo estigma das lesões pigmentadas quanto pelas inúmeras internações e procedimentos que se tornam necessários até a excisão total da lesão. Para evitar sequelas psicológicas e degenerações malignas, tem se optado por iniciar o mais precocemente o tratamento cirúrgico dessas crianças, com o objetivo de terminar o tratamento em idade pré-escolar, se possível.

\section{REFERÊNCIAS}

1. Heffek DF, Thaller S. Congenital melanosis: an update. J Craniofac Surg. 2005;16(5):940-4.

2. Bentz ML, Bauer BS, Zuker RM. Principles and practice of pediatric plastic surgery. St Louis:Quality Medical Publishing;2007.

3. Castilla EE, Dutra MG, Oriolo-Parreiras IM. Epidemiology of congenital pigmented naevi: incidence rates relative frequencies. Br J Dermatol. 1981;104(3):307-15.

4. Arneja JS, Gosain AK. Giant congenital melanocytic nevi. Plast Reconstr Surg. 2007;120(2):26e-40e.

5. Turkmen A, Isik D, Bekerecioglu M. Comparison of classifica- tion systems for congenital melanocytic nevi. Dermatol Surg. 2010;36(10):1554-62.

6. Kaplan E, Nickoloff BJ. Clinical and histologic features of nevi with emphasis on treatment approaches. Clin Plast Surg. 1987;14(2):277300 .

7. Quaba AA, Wallace AF. The incidence of malignant melanoma (0 to 15 years of age) arising in "large" congenital nevocellular nevi. Plast Reconstr Surg 1986;78(2):174-81.

8. Bauer BS, Corcoran J. Treatment of large and giant nevi. Clin Plast Surg. 2005;32(1):11-8.

9. Chung C, Forte AJ, Narayan D, Persing J. Giant nevi: a review. J Craniofac Surg. 2006;17(6):1210-5.

10. Solomon LM. The management of congenital melanocytic nevi. Arch Dermatol. 1980;116(9):1017.

11. Kaplan EN. The risk of malignancy in large congenital nevi. Plast Reconstr Surg. 1974;53(4):421-8.

12. Watt AJ, Kotsis SV, Chung KC. Risk of melanoma arising in large congenital melanocytic nevi: a systematic review. Plast Reconstr Surg. 2004;113(7):1968-74.

13. Rhodes AR, Wood WC, Sober AJ, Mihm MC Jr. Nonepidermal origin of malignant melanoma associated with a giant congenital nevocellular nevus. Plast Reconstr Surg. 1981;67(6):782-90.

14. Trozak DJ, Rowland WR, Hu F. Metastatic malignant melanoma in prepubertal children. Pediatrics. 1975;55(2):191-204.

15. Arneja JS, Gosain AK. Giant congenital melanocytic nevi of the trunk and an algorithm for treatment. J Craniofac Surg. 2005;16(5):886-93.

16. Elias DL, Baird WL, Zubowicz VN. Applications and complications of tissue expansion in pediatric patients. J Pediatr Surg. 1991;26(1):15-21.

17. Iconomou TG, Michelow BJ, Zuker RM. Tissue expansion in the pediatric patient. Ann Plast Surg. 1993;31(2):134-40.

18. Gibstein LA, Abramson DL, Bartlett RA, Orgill DP, Upton J, Mulliken JB. Tissue expansion in children: a retrospective study of complications. Ann Plast Surg. 1997;38(4):358-64.

19. Neale HW, High RM, Billmire DA, Carey JP, Smith D, Warden G. Complications of controlled tissue expansion in the pediatric burn patient. Plast Reconstr Surg. 1998;82(5):840-8.

20. Hurvitz KA, Rosen H, Meara JG. Pediatric cervicofacial tissue expansion. Int J Pediatr Otorhinolaryngol. 2005;69(11):1509-13.

21. Adler N, Dorafshar AH, Bauer BS, Hoadley S, Tournell M. Tissue expander infections in pediatric patients: management and outcomes. Plast Reconstr Surg. 2009;124(2):484-9.

22. Danarti R, Konig A, Happle R. Large congenital melanocytic nevi may reflect paradominant inheritance implying allelic loss. Eur J Dermatol. 2003;13(5):430-2.

23. Keipert JA. Giant pigmented naevus: the frequency of malignant change and indications for treatment in prepubertal children. Australas J Dermatol. 1985;26(2):81-5.

24. Bergfeld W, Helwig WB. Risk of developing melanoma in congenital nevi. Presented at the Annual Meeting of the American Academy of Dermatology, Miami, FL, December 27, 1972.

25. Shah KN. The risk of melanoma and neurocutaneous melanosis associated with congenital melanocytic nevi. Semin Cutan Med Surg. 2010;29(3):159-64.

26. Marghoob AA, Dusza S, Oliveria S, Halpern AC. Number of satellite nevi as a correlate for neurocutaneous melanocytosis in patients with large congenital melanocytic nevi. Arch Dermatol. 2004;140(2):171-5.

27. Kadonaga JN, Frieden IJ. Neurocutaneous melanosis: definition and review of literature. J Am Acad Dermatol. 1991;24(5 Pt 1):747-55.

Correspondência para: 